\title{
Terapia complementar de reiki nos fatores associados à qualidade de vida em pacientes diagnosticados com câncer: uma revisão sistemática
}

\author{
Complementary therapy of reiki on the quality of life in patients diagnosed with cancer: a \\ systematic review
}

Terapia reiki adicional en factores de calidad de vida en pacientes de diagnóstico de cáncer: una revisión sistemática

Victor Hugo Dantas Guimarães ${ }^{1 *}$, Ana Luíza Barbosa de Souza', Jaciara Neves Sousa', Bruna Matos Gusmão ${ }^{1}$, Amanda Mota Lacerda ${ }^{1}$, João Lucas Rodrigues dos Santos ${ }^{1}$, Daniela Fernanda de Freitas ${ }^{2}$.

\section{RESUMO}

Objetivo: Avaliar através de uma revisão a influência da terapia de Reiki na qualidade de vida de pacientes diagnosticados com câncer. Métodos: Este estudo seguiu os padrões estabelecidos pelos principais itens para relatar Revisões sistemáticas e Meta-análises (PRISMA), utilizando as bases de dados PubMed, EBSCO e Web of Science. Resultados: Foram identificados 175 artigos, sendo destes 21 artigos selecionados para incluir a revisão. Dos artigos utilizados, 5 apresentaram alto nível (II) de evidencia, caracterizados por ensaios randomizados e controlados; 5 artigos apresentaram nível IV, caso-controle; 3 estudos com o nível III (ensaio randomizado); e outros 3 artigos sem nível classificação de evidência. Os estudos evidenciaram melhoras em variáveis como: vigor, fadiga dor, humor e náuseas. As melhoras desses parâmetros foram corroboradas pelas análises do tamanho do efeito. Algumas limitações do estudo consistiram em grande variabilidade dos tipos de cancer, tamanho amostral pequeno, delineamento dos estudos e o uso do termo técnico empregado para terapia que difilcultam as comparações entre os estudos. Considerações finais: A aplicação do Reiki torna-se uma ferramenta alternativa importante coadjuvante para melhora da qualidade de vida em pacientes com câncer, apesar da limitada quantidade de artigos com alta qualidade metodológica.

Palavras-chave: Neoplasias, Toque terapêutico, Qualidade de vida.

\begin{abstract}
Objective: To evaluate, through a review, the influence of Reiki therapy on the quality of life of patients diagnosed with cancer. Methods: This study followed the standards established by the main items for reporting Systematic Reviews and Meta-analyzes (PRISMA), using the PubMed, EBSCO and Web of Science databases. Results: 175 articles were identified, of which 21 were selected to include the review. Of the articles used, 5 had a high level (II) of evidence, characterized by randomized controlled trials; 5 articles presented level IV, case-control; 3 studies with level III (randomized trial); and another 3 articles with no evidence classification level. Studies have shown improvements in variables such as: vigor, fatigue, pain, mood and nausea. The improvements in these parameters were corroborated by the effect size analyzes. Some limitations of the study consisted of great variability in the types of cancer, small sample size, design of the studies and the use of the technical term used for therapy that make comparisons between studies difficult. Final considerations: The application of Reiki becomes an important alternative supporting tool for improving the quality of life in cancer patients, despite the limited amount of articles with high methodological quality.
\end{abstract}

Keywords: Neoplasms, Therapeutic touch, Quality of life.

\footnotetext{
${ }^{1}$ Universidade Estadual de Montes Claros (Unimontes). Montes Claros - MG.

*E-mail: guimarães.vhd@gmail.com

${ }^{2}$ Faculdades Santo Agostinho (FASA). Montes Claros - MG.
}

SUBMETIDO EM: 5/2020

ACEITO EM: 5/2020

PUBLICADO EM: $8 / 2020$ 


\section{RESUMEN}

Objetivo: evaluar, a través de una revisión, la influencia de la terapia de Reiki en la calidad de vida de los pacientes diagnosticados con cáncer. Métodos: Este estudio siguió los estándares establecidos por los ítems principales para reportar Revisiones Sistemáticas y Metaanálisis (PRISMA), utilizando las bases de datos PubMed, EBSCO y Web of Science. Resultados: se identificaron 175 artículos, de los cuales 21 fueron seleccionados para incluir la revisión. De los artículos utilizados, 5 tenían un alto nivel (II) de evidencia, caracterizado por ensayos controlados aleatorios; 5 artículos presentaron nivel IV, control de casos; 3 estudios con nivel III (ensayo aleatorio); y otros 3 artículos sin nivel de clasificación de evidencia. Los estudios han demostrado mejoras en variables como: vigor, fatiga, dolor, estado de ánimo y náuseas. Las mejoras en estos parámetros fueron corroboradas por los análisis del tamaño del efecto. Algunas limitaciones del estudio consistieron en una gran variabilidad en los tipos de cáncer, el pequeño tamaño de la muestra, el diseño de los estudios y el uso del término técnico utilizado para la terapia que dificulta las comparaciones entre los estudios. Consideraciones finales: la aplicación de Reiki se convierte en una importante herramienta de apoyo alternativa para mejorar la calidad de vida en pacientes con cáncer, a pesar de la cantidad limitada de artículos con alta calidad metodológica

Palabras clave: Neoplasias, Tacto terapéutico, Cualidad de vida.

\section{INTRODUÇÃO}

O Câncer é caracterizado pelo crescimento desordenado de células que invadem os tecidos e órgãos, podendo espalhar-se para outras regiões do corpo. Este pode originar-se em detrimento de vários fatores, genético e/ou epigenéticos (POPAT S, 2005). Diferentes tipos de câncer distinguem entre si pela velocidade de multiplicação das células e a capacidade de invadir tecidos e órgãos vizinhos ou distantes, denominado metástase. No Brasil, estima-se que para cada ano do triênio 2020-2022 aponta que ocorrerão 625 mil casos novos de câncer (450 mil, excluindo os casos de câncer de pele não melanoma). O câncer de pele não melanoma consistirá no mais incidente (177 mil), seguido pelos cânceres de mama e próstata (66 mil cada), cólon e reto ( $41 \mathrm{mil})$, pulmão (30 mil) e estômago (21 mil) (MARTIN TA, et al., 2013; MATHERS CD, et al., 2003).

Um fator relevante que tem contribuído para o aumento da sobrevida dos pacientes com câncer são os avanços da medicina, permitindo tratamentos mais eficazes e técnicas que possibilitam a detecção precoce (VENÂNCIO JL, 2004). Segundo o Instituto Nacional do Câncer (INCA), o tratamento do câncer pode ser feito por meio de cirurgia, radioterapia, quimioterapia ou transplante de medula óssea. Em muitos casos, é necessário combinar mais de uma modalidade (INCA, 2018). O diagnóstico e tratamento do câncer têm sido visto como um processo doloroso vivido pelo paciente e sua a família. Nesse sentindo tratamentos alternativos e/ou complementares estão sendo utilizados cada vez mais a fim de minimizar sofrimentos e incrementar a restauração da saúde (ALARCÃO Z e FONSECA JR, 2016).

Mediante os avanços advindos de novas tecnologias implementadas, a sobrevida em pacientes diagnosticados com neoplasias tem crescido substancialmente, o que tem direcionado investimentos à criação de programas de saúde para a promoção da qualidade de vida destes pacientes (BLOOM JR, 2002). Em geral, a qualidade de vida é definida como um conceito multidimensional, qual avalia subjetivamente 0 estado de bem-estar e saúde por fatores físicos e psíquicos (PARKER PA, et al., 2003).

O uso de medicina alternativa complementar nos sistemas nacionais de saúde tem sido objeto de constante debate. Existem importantes documentos de referência da Organização Mundial da Saúde que defendem a necessidade de pesquisas sobre medicamentos tradicionais e complementares: (I) políticas nacionais para incluir sua prática nos sistemas nacionais de saúde, (II) segurança, eficácia e qualidade dessas práticas, (III) acesso a elas e (IV) uso racional por profissionais e consumidores (WORD HEALTH ORGANIZATION 2018; LARCÃO Z e FONSECA JR, 2016). 
O Reiki é uma terapia complementar derivada das tradições de saúde e cura oriental. A prática foi desenvolvida em 1922 pelo monge budista japonês Mikao Usui. É fundamentado sobre o "fluxo de uma forma psíquica de energia", simbolizada em japonês por ki, manipulável através da imposição de mãos. Recentemente foi incluída entre os programas terapêuticos complementares derivados da saúde oriental e tradições de cura para lidar com o câncer em ambientes hospitalares (JOYCE J e HERBISON GP, 2015; RAND WL, 1995). Evidências na literatura demonstram que o Reiki pode ser uma ferramenta benéfica no tratamento do câncer. Atua na melhora do sono, sensação de calma e paz, redução da depressão, melhorando também, a autoconfiança após intervenção com sessões de Reiki, além de proporcionar, relaxamento, esperança, melhorias físicas, emocionais e cognitivas (DEMIR M, et al., 2015; KIRSHBAUM; STEAD H e BARTYS MN, 2016).

Em suma, ainda não está totalmente claro o efeito do Reiki em pacientes com câncer. Portanto, o presente estudo objetivou revisar sistematicamente a literatura sobre a aplicação do Reiki e seus fatores associados a qualidade de vida de pacientes diagnosticados com câncer.

\section{MÉTODOS}

Este estudo seguiu os padrões estabelecidos pelos principais itens para relatar Revisões sistemáticas e Meta-análises (PRISMA) (LIBERATI A, et al., 2009).

\section{Critérios de elegibilidade dos estudos}

Realizou-se uma busca sem delimitação de tempo ou idioma, bem como ensaios de intervenções clínicas, contemplando artigos originais que investigaram o efeito da terapia Reiki em fatores associados à qualidade de vida de pessoas diagnosticadas com câncer.

\section{Critério de exclusão}

Os estudos não foram inclusos devido: (1) Ausência de evidências intervencionais (por exemplo: implementação da terapia de Reiki em acompanhantes de pacientes internos com câncer); (2) Combinar intervenções (por exemplo: tratamento com Reiki e yoga, "passe espírita", medicina complementar alternativa - CAM); (3) Revisão de literatura de qualquer natureza; (4) Projetos de estruturação curricular integralholístico.

\section{Recursos}

Os artigos foram pesquisados nas bases de dados PubMed, Business Source Complete (EBSCO) e Web of Science. Além das bases de dados descritas, foram recuperados artigos em listas de referências de outros artigos originais relacionados ao tema.

\section{Estratégia de pesquisa}

Para buscar os artigos, utilizou-se uma combinação dos seguintes termos: Neoplasia, Cancer, Tumor, Therapeutic Touch, Reiki, Laying-on-of-Hands. Todos os termos foram identificados no título ou nos resumos dos artigos usando a estratégia (Patient, Intervention, Comparison and Outcome) PICOS (SCHARDT C, et al., 2007). Recursos adicionais foram utilizados de acordo com a disponibilidade do banco de dados (por exemplo, Medical Subject Headings - MeSH) para amplificar a busca. A estratégia de busca realizada no PubMed é listada a seguir: (((cancer[Title/Abstract]) OR tumor[Title/Abstract]) OR neoplasia[Title/Abstract])) AND (((reiki[Title/Abstract]) OR Laying-on-of-Hands[Title/Abstract]) OR Touch, Therapeutic[Title/Abstract]). Este procedimento teve como objetivo não só filtrar os resultados, mas também cruzar os principais termos para obter o máximo de estudos possíveis.

\section{Seleção de estudo}

A seleção dos artigos respeitou três etapas, realizadas por dois revisores: 1) leitura dos títulos; 2) leitura dos resumos; 3) leitura de texto na íntegra. Apenas títulos que continham pelo menos três dos termos supracitados (therapeutic touch/reiki/cancer) ou seus sinônimos foram aceitos. Em seguida, o resumo foi lido para identificar o tipo de intervenção e os desfechos (qualidade de vida, estresse, fadiga, vigor, humor e dor). Artigos duplicados em bancos de dados e não recuperados em banco de dados foram excluídos. 


\section{Níveis de evidências de estudos individuais}

Utilizou-se a escala Prática Baseada em Evidências (BPE) (FINEOUT-OVERHOLT E, et al., 2010) para classificar os estudos de acordo com a qualidade metodológica, a fim de expor possíveis vieses de falhas metodológicas em tais artigos.

\section{RESULTADOS E DISCUSSÃO}

A busca sumarizou o total de 175 estudos, sendo um artigo não recuperado da base de dados PubMed, embora tenha sido realizado o contato com os respectivos autores do trabalho. Após os critérios de seleção, 21 estudos foram inclusos para compor a revisão (Figura 1).

Figura 1 - Fluxograma de triagem, inclusão, exclusão e seleção de estudos.

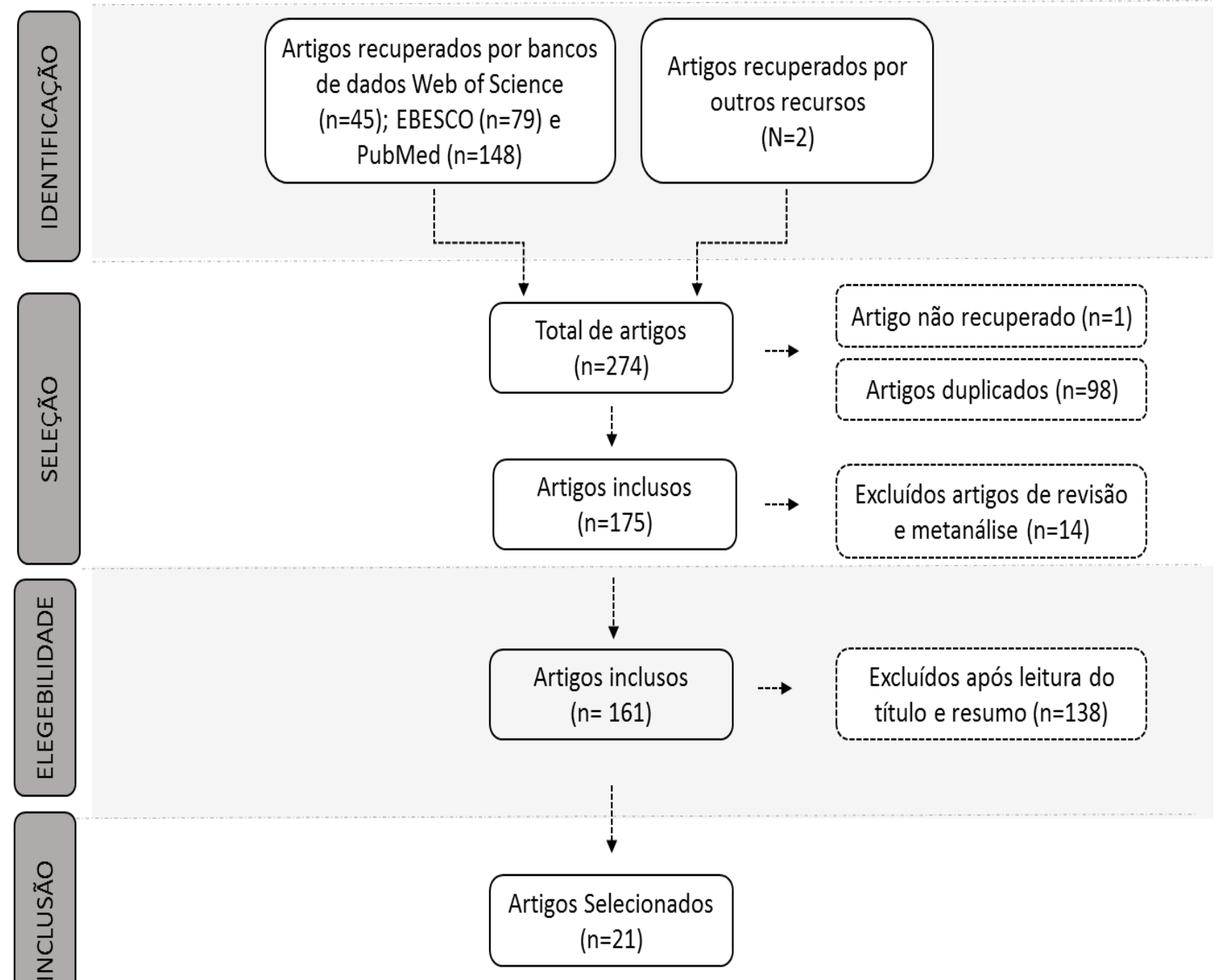

Fonte: Guimarães VHD, et al., 2020.

A quimioterapia é um dos tratamentos contra o câncer mais antigo e mais comum em todo o mundo, realizada em cerca de $70-80 \%$ dos pacientes. Entre os efeitos colaterais mais graves e temidos pelos mesmos, estão a náusea e o vômito induzidos por esse tipo de terapêutica. Esses sintomas contribuem para a redução da qualidade de vida e declínio na realização das atividades de vida diária desses pacientes e podem, ainda, diminuir a adesão ao tratamento (VANAKI Z, et al., 2016). 
Diante dos efeitos colaterais do tratamento do câncer, a Medicina Complementar apresenta-se, como uma opção da melhora da qualidade de vida para esses pacientes. O Reiki é um sistema de cura natural, realizado através da colocação das mãos e da transferência de energia do praticante para o destinatário.

Acredita-se que a sua capacidade de cura acontece através do reequilíbrio de energia e, assim, restaura o bem-estar físico, emocional, mental e espiritual do paciente. Entre as técnicas de relaxamento, para melhorar o enfrentamento com câncer, Reiki tem se mostrado eficaz para aliviar a dor e outros sintomas como ansiedade, insônia, hiporexia, aumentando assim a qualidade de vida dos pacientes com câncer avançado, como demonstrado na tabela 1.

Esses relatos foram demonstrados através de uma pesquisa desenvolvida por Birocco N, et al. (2012), em que a média do escore de ansiedade teve uma redução estatisticamente significativa. A terapia Reiki não possui efeitos colaterais e é muito apreciada por todos os pacientes. No geral, as sessões foram consideradas positivas para melhorar o bem-estar (70\%), relaxamento (88\%), alívio da dor (45\%), qualidade do sono (34\%) e na redução da ansiedade (70\%) (BIROCCO N, et al., 2012). 


\section{Revista Eletrônica Acervo Saúde / Electronic Journal Collection Health ISSN 2178-2091}

Tabela 1 - Evidências da terapia de Reiki em diferentes parâmetros da qualidade de vida em pacientes diagnosticados com câncer.

\begin{tabular}{|c|c|c|c|c|c|c|}
\hline Autor (Ano) & Amostra & NE & $\begin{array}{c}\text { Frequência } \\
\text { (Semanal) }\end{array}$ & Semanas & Instrumentos utilizados & Resultados \\
\hline $\begin{array}{l}\text { YOUNUS J, } \\
\text { et al., } 2015\end{array}$ & 49 Mulheres & IV & $\begin{array}{c}3 \text { vezes } \\
(15 \text { a } 20 \mathrm{~min})\end{array}$ & 5 & $\begin{array}{l}\text { Escala de dermatite (Toxicity was } \\
\text { assessed using dermatitis scale) NCIC } \\
\text { CTC V3, classificação cosmética EORTC } \\
\text { Breast Cosmetic Rating e a qualidade de } \\
\text { vida, humor, energia e fadiga foram } \\
\text { avaliadas pela escalas quality of } \\
\text { life (QLQ C30), Profile of Mood States } \\
\text { (POMS) e (Brief Fatigue Inventory) BFI. }\end{array}$ & Não foi possível detectar um benefício significativo \\
\hline $\begin{array}{l}\text { KIRSHBAU } \\
\text { MN, et al., } \\
2016 \\
\end{array}$ & $\begin{array}{c}10 \text { mulheres } \\
\text { A idade média foi de } 63 \text { anos } \\
\text { (variação de } 31 \text { a } 84 \text { anos) }\end{array}$ & VI & (20 a 60 min) & $\sim 5$ & $\begin{array}{l}\text { Entrevistas transcritas e codificadas. } \\
\text { Análise do framework }\end{array}$ & $\begin{array}{c}\text { O reiki pode ser uma ferramenta benéfica na } \\
\text { autogestão dos problemas de qualidade de vida das } \\
\text { mulheres com câncer. }\end{array}$ \\
\hline $\begin{array}{l}\text { CHARLESW } \\
\text { ORTH E, et } \\
\text { al., } 2018\end{array}$ & $\begin{array}{l}74 \text { mulheres, } 22 \text { homens. } \\
\text { Idade média foi de } 54,92 \\
\text { (variação de } 26 \text { a } 83 \text { anos) }\end{array}$ & - & 4 & $\begin{array}{l}\text { Variações } \\
8, \sim 11 \\
\text { semanas e } \\
1 \text { ano. } \\
\end{array}$ & $\begin{array}{l}\text { Ferramenta de avaliação MyCaW (Medir } \\
\text { suas preocupações e bem-estar) }\end{array}$ & $\begin{array}{l}\text { As terapias complementares demonstraram melhorias } \\
\text { significativas, as maiores melhorias foram vistas em } \\
\text { relaxamento, problemas de sono e dores. }\end{array}$ \\
\hline $\begin{array}{l}\text { DEMIR M, et } \\
\text { al., } 2015\end{array}$ & $\begin{array}{l}\text { Mulheres }(71,4 \%) \text {, indivíduos } \\
\text { casados ( } 40 \%) \text { e graduados } \\
\text { do ensino fundamental ( } 40 \%)\end{array}$ & II & 5 (30 min) & SI & $\begin{array}{l}\text { Entrevista face a face usando o } \\
\text { formulário Patient Characteristics. }\end{array}$ & $\begin{array}{c}\text { Os resultados deste estudo indicam que o Reiki pode } \\
\text { diminuir a dor, a ansiedade e a fadiga em pacientes } \\
\text { oncológicos. }\end{array}$ \\
\hline $\begin{array}{l}\text { FLEISHER } \\
\text { KA, et al., } \\
2014\end{array}$ & $\begin{array}{c}\text { Das } 213 \text { sessões, } 136(63,9 \%) \\
\text { foram realizadas em mulheres, } \\
50(23,5 \%) \text { em homens e } 27 \\
(12,7 \%) \text { em sujeitos de gênero } \\
\text { não declarado. }\end{array}$ & - & $\begin{array}{c}\text { Mediana de } 2 \\
\text { sessões com } \\
\text { intervalo de } 2 \text { a } \\
11 . \\
10 \text { a } 30 \text { min }\end{array}$ & $\begin{array}{l}\text { Programa } \\
\text { de } \\
\text { Voluntariad } \\
\text { o do Reiki. }\end{array}$ & $\begin{array}{l}\text { Um instrumento de pesquisa } \\
\text { Projetado pelo autor, além de uma } \\
\text { versão modificada do Distress } \\
\text { Termômetro (DT) }\end{array}$ & $\begin{array}{l}\text { Um programa de voluntariado integrativo de Reiki se } \\
\text { mostra promissor como um componente do cuidado de } \\
\text { suporte para pacientes com câncer. } • \text { O Reiki } \\
\text { apresenta benefícios positivos a curto prazo, } \\
\text { especialmente atendendo às necessidades espirituais } \\
\text { e emocionais dos pacientes com câncer. Além disso, } \\
\text { pode ativar a resposta de relaxamento. }\end{array}$ \\
\hline $\begin{array}{l}\text { ORSAK G, } \\
\text { et al., } 2015\end{array}$ & $\begin{array}{c}36 \text { pacientes (35 mulheres, } 1 \\
\text { homem) } 34 \text { e } 67 \text { anos de } \\
\text { idade. } \\
35 \text { pacientes eram americanos } \\
\text { europeus }(97,3 \%) \text { e } 1 \text { era afro- } \\
\text { americano }(2,7 \%) \text {. }\end{array}$ & II & $30 \mathrm{~min}$ & SI & $\begin{array}{l}\text { A qualidade de vida multidimensional foi } \\
\text { medida usando a Escala de Avaliação } \\
\text { Funcional de Terapia do Câncer: Câncer } \\
\text { de Mama Versão 4, 17. • Angústia } \\
\text { relacionada aos sintomas do câncer (por } \\
\text { exemplo, náusea) foi medido utilizando- } \\
\text { se a escala Symptom Distress Scale.18. } \\
\text { - Os estados de humor foram avaliados } \\
\text { usando o questionário Perfil dos Estados } \\
\text { de Humor - Questionário de Forma } \\
\text { Curta.19. - Questões relacionadas à } \\
\text { terapia de Reiki. }\end{array}$ & $\begin{array}{c}\text { O Reiki foi classificado como relaxante, sem efeitos } \\
\text { colaterais. Reiki e grupos acompanhantes relataram } \\
\text { melhorias na qualidade de vida e humor que foram } \\
\text { maiores do que as observadas no grupo de cuidados } \\
\text { habituais. Intervenções durante a quimioterapia, como } \\
\text { Reiki ou companheirismo, são viáveis, aceitáveis e } \\
\text { podem reduzir os efeitos colaterais. }\end{array}$ \\
\hline
\end{tabular}

REAS/EJCH | Vol.12(12) | e3797 | DOI: https://doi.org/10.25248/reas.e3797.2020 Página 6 de 14 


\section{Revista Eletrônica Acervo Saúde / Electronic Journal Collection Health ISSN 2178-2091}

\begin{tabular}{|c|c|c|c|c|c|c|}
\hline $\begin{array}{l}\text { ALARCÃO Z } \\
\text { e FONSECA } \\
\text { JR, et al., } \\
\quad 2016\end{array}$ & $\begin{array}{l}58 \text { pacientes do sexo } \\
\text { masculino }\end{array}$ & II & $\begin{array}{l}2 \text { vezes } \\
60 \mathrm{~min}\end{array}$ & 4 & $\begin{array}{l}\text { A qualidade de vida foi medida usando a } \\
\text { versão em português do World Health } \\
\text { Organization quality of Life - Brief } \\
\text { (WHOQoL-Bref). }\end{array}$ & $\begin{array}{l}\text { O Reiki demonstrou melhorias nas dimensões geral, } \\
\text { física, ambiental e social. Estes resultados sugerem } \\
\text { que o Reiki pode ser uma opção eficaz e segura para } \\
\text { melhorar o bem-estar em pacientes com câncer no } \\
\text { sangue. Esses achados podem dar suporte à inclusão } \\
\text { do Reiki nos serviços nacionais de saúde, uma vez que } \\
\text { isso pode fornecer uma contribuição útil para melhorar } \\
\text { o bem-estar dos pacientes e, consequentemente, uma } \\
\text { melhor qualidade de vida. }\end{array}$ \\
\hline $\begin{array}{l}\text { OLSON K, et } \\
\text { al., } 2003\end{array}$ & $\begin{array}{l}24 \text { pacientes adultos ( } 9 \\
\text { homens, } 15 \text { mulheres). }\end{array}$ & IV & $\begin{array}{l}\text { Participantes } \\
\text { (uma hora após a } \\
\text { primeira dose } \\
\text { analgésica da } \\
\text { tarde. Os do } \\
\text { (braço B) } \\
\text { receberam um } \\
\text { tratamento de } \\
\text { Reiki nos dias } 1 \text { e } \\
4,1 \text { hora após a } \\
\text { primeira dose } \\
\text { analgésica da } \\
\text { tarde. Braço A) } \\
\text { descansaram por } \\
1,5 \text { hora nos dias } \\
1 \text { e } 4\end{array}$ & SI & $\begin{array}{l}\text { A medida de qualidade de vida foi uma } \\
\text { ferramenta multidimensional com } \\
\text { subescalas físicas, sociais e } \\
\text { psicológicas. A validade de construto } \\
\text { dessa ferramenta foi estabelecida } \\
\text { através de análise fatorial e coeficientes } \\
\text { foram pelo menos } 0,65 \text { em estudos com } \\
\text { pacientes com câncer. }\end{array}$ & $\begin{array}{l}\text { Os participantes que receberam terapia opioide padrão } \\
\text { mais Reiki relataram melhora significativa na dor, } \\
\text { queda na pressão arterial diastólica e no pulso, bem } \\
\text { como, melhora significativa no componente psicológico } \\
\text { da qualidade de vida. }\end{array}$ \\
\hline $\begin{array}{l}\text { GIASSON M } \\
\text { e } \\
\text { BOUCHARD } \\
\text { L, } 1998\end{array}$ & $\begin{array}{l}20 \text { pessoas com câncer } \\
\text { terminal em cuidados } \\
\text { paliativos. }\end{array}$ & IV & $\begin{array}{l}15 \text { a } 20 \text { minutos } \\
\text { (Grupo } \\
\text { experimental) } \\
\text { Três períodos de } \\
\text { descanso (Grupo } \\
\text { controle) }\end{array}$ & $\begin{array}{l}4 \text { dias } \\
\text { consecutiv } \\
\text { os }\end{array}$ & $\begin{array}{c}\text { O bem-estar foi medido no tempo de pré- } \\
\text { intervenção e imediatamente após a } \\
\text { intervenção usando a Escala de Bem- } \\
\text { Estar, uma escala analógica visual que } \\
\text { mede dor, náusea, depressão, } \\
\text { ansiedade, falta de ar, atividade, apetite, } \\
\text { relaxamento e paz interior. }\end{array}$ & $\begin{array}{l}\text { Os resultados do estudo apóiam a hipótese de que três } \\
\text { tratamentos com Toque Terapêutico sem contato } \\
\text { aumentam a sensação de bem-estar em pessoas com } \\
\text { câncer terminal. }\end{array}$ \\
\hline $\begin{array}{l}\text { BEARD C, et } \\
\text { al., } 2011\end{array}$ & 18 homens & II & $\begin{array}{l}\text { Duas vezes por } \\
\text { semana, duraram } \\
\text { aproximadamente } \\
50 \text { minutos. }\end{array}$ & $\begin{array}{l}8 \text { semanas } \\
\text { consecutiv } \\
\text { as para um } \\
\text { total } \\
\text { máximo de } \\
16 \\
\text { sessões. }\end{array}$ & $\begin{array}{l}\text { Instrumentos bem validados mediram a } \\
\text { ansiedade (STAI), depressão (CES-D) e } \\
\text { qualidade de vida em pacientes com } \\
\text { câncer (FACT-G). Inventário de } \\
\text { Ansiedade do Estado de Spielberger } \\
\text { (STAI); Escala Centro de Depressão de } \\
\text { Estudos Epidemiológicos (CES-D); } \\
\text { Escala de avaliação Funcional da } \\
\text { Terapia Geral contra o Câncer (FACT-G). }\end{array}$ & $\begin{array}{c}15 \text { de } 18(83 \%) \text { pacientes Reiki completaram o } \\
\text { protocolo de intervenção. Nenhuma diferença } \\
\text { estatisticamente significativa foi encontrada entre os } \\
\text { grupos RRT, Reiki e controle nas pontuações totais } \\
\text { para os instrumentos STAI, CES-D ou FACT-G em } \\
\text { qualquer momento. No entanto, os participantes que } \\
\text { foram classificados como "ansiosos" no início do } \\
\text { estudo, houve uma tendência positiva foi encontrada } \\
\text { no grupo Reiki. }\end{array}$ \\
\hline
\end{tabular}

REAS/EJCH | Vol.12(12) | e3797 | DOI: https://doi.org/10.25248/reas.e3797.2020 Página 7 de 14 


\section{Revista Eletrônica Acervo Saúde / Electronic Journal Collection Health ISSN 2178-2091}

\begin{tabular}{|c|c|c|c|c|c|c|}
\hline $\begin{array}{l}\text { LAFRENIER } \\
\text { E KD, et al., } \\
1999\end{array}$ & $\begin{array}{l}41 \text { voluntárias saudáveis do } \\
\text { sexo feminino. }\end{array}$ & III & $\begin{array}{c}\text { Descanso por um } \\
\text { período de } 5 \text { a } 10 \\
\text { minutos. }\end{array}$ & $\begin{array}{l}\text { Três } \\
\text { sessões } \\
\text { mensais. }\end{array}$ & $\begin{array}{l}\text { Depois do período de descanso, as } \\
\text { participantes foram acompanhadas a } \\
\text { uma sala de testes nas proximidades, } \\
\text { onde preencheu o questionário. }\end{array}$ & $\begin{array}{l}\text { Os resultados indicaram que o transtorno do humor no } \\
\text { grupo experimental diminuiu significativamente ao } \\
\text { longo das três sessões, enquanto o grupo controle } \\
\text { aumentou em distúrbios do humor ao longo do } \\
\text { tempo. Especificamente, os participantes do grupo } \\
\text { experimental mostraram reduções significativas de } \\
\text { tensão, confusão, e ansiedade e um aumento } \\
\text { significativo no vigor em todas as sessões. }\end{array}$ \\
\hline $\begin{array}{l}\text { POHL G, et } \\
\text { al., } 2007\end{array}$ & 80 pacientes. & III & $5 \mathrm{~min}$. & $\begin{array}{l}\text { Três vezes } \\
\text { por } \\
\text { semana. }\end{array}$ & $\begin{array}{l}\text { O efeito do tratamento foi medido usando } \\
\text { uma "escala de bem-estar", com a } \\
\text { diferença da pontuação média da "escala } \\
\text { de bem-estar" no dia } 10 \text { sendo definida } \\
\text { como primária e que no dia } 5 \text { como } \\
\text { desfecho secundário. }\end{array}$ & $\begin{array}{l}\text { "Colocação das mãos" resultou em uma melhora } \\
\text { significativa dos sintomas associados à terapia de } \\
\text { câncer. A magnitude da melhora obtida foi semelhante, } \\
\text { seja em um "tratamento" auto-declarado-curandeiro ou } \\
\text { um "tratamento" fornecido pelo ator. }\end{array}$ \\
\hline $\begin{array}{l}\text { TSANG KL, } \\
\text { et al., } 2007\end{array}$ & $\begin{array}{c}6 \text { pacientes ( } 13 \text { mulheres e } 3 \\
\text { homens). }\end{array}$ & III & 45 minutos. & $\begin{array}{l}5 \text { dias } \\
\text { consecutiv } \\
\text { os. }\end{array}$ & $\begin{array}{l}\text { Questionários: Avaliação Funcional da } \\
\text { Subescala Fadiga do Câncer [FACT-F]); } \\
\text { Avaliação Funcional da Terapia do } \\
\text { Câncer, Versão Geral [FACT-G]). } \\
\text { Também completaram uma escala } \\
\text { analógica visual (Edmonton Symptom } \\
\text { Assessment System [ESAS])) antes e } \\
\text { depois de cada sessão de tratamento. }\end{array}$ & $\begin{array}{c}\text { A fadiga no FACT-F diminuiu dentro da condição do } \\
\text { Reiki ao longo de todos os } 7 \text { tratamentos. Além disso, } \\
\text { os participantes da condição de Reiki experimentaram } \\
\text { melhorias significativas na qualidade de vida (FACT-G) } \\
\text { em comparação com aqueles na condição de } \\
\text { repouso. Em avaliações diárias (ESAS) na condição de } \\
\text { Reiki, as pontuações da precisão } 1 \text { versus pós-sessão } \\
5 \text { indicaram reduções significativas no cansaço, dor e } \\
\text { ansiedade, que não foram vistas na condição de } \\
\text { repouso. }\end{array}$ \\
\hline $\begin{array}{l}\text { POST- } \\
\text { WHITE J, et } \\
\text { al., } 2003\end{array}$ & 230 indivíduos & & $\begin{array}{l}\text { Todos os sujeitos } \\
\text { receberam } \\
\text { sessões semanais } \\
\text { de } 45 \text { minutos de } \\
\text { intervenção } \\
\text { designada (MT, } \\
\text { HT ou P) e } 4 \\
\text { sessões semanais } \\
\text { de um tratamento } \\
\text { / controle padrão. }\end{array}$ & 4 semanas & $\begin{array}{c}\text { Índice Breve de Dor (BPI), Índice Breve } \\
\text { de Náusea (BNI) e fadiga, ansiedade e } \\
\text { perturbação do humor, conforme medido } \\
\text { pelo Perfil dos Estados de Humor } \\
\text { (POMS). }\end{array}$ & $\begin{array}{l}\text { A massagem terapêutica (MT) e do toque de cura (HT), } \\
\text { diminuíram a pressão arterial, frequência respiratória } \\
\text { (RR) e frequência cardíaca (FC). MT reduziu a } \\
\text { ansiedade e HT diminuiu a fadiga, e ambos reduziram } \\
\text { a perturbação total do humor. MT e HT são mais } \\
\text { eficazes do que a presença isolada ou tratamento } \\
\text { padrão na redução da dor, distúrbios de humor e fadiga } \\
\text { em pacientes que recebem quimioterapia para câncer. }\end{array}$ \\
\hline
\end{tabular}

REAS/EJCH | Vol.12(12) | e3797 | DOI: https://doi.org/10.25248/reas.e3797.2020 Página 8 de 14 


\section{Revista Eletrônica Acervo Saúde / Electronic Journal Collection Health ISSN 2178-2091}

\begin{tabular}{|c|c|c|c|c|c|c|}
\hline $\begin{array}{l}\text { SIEGEL P, } \\
\text { et al., } 2016\end{array}$ & 36 pacientes & IV & 5 vezes $-30 \mathrm{~min}$ & 1 semana & $\begin{array}{l}\text { Questionário MYMOP (atividade e nível } \\
\text { de bem-estar). Questionário adaptado } \\
\text { Measure Yourself Medical Outcome } \\
\text { Profile (av-MYMOP) permite ao paciente } \\
\text { elaborar sua experiência da doença, os } \\
\text { sintomas e os níveis de bem-estar. } \\
\text { No caso de } 5 \text { pacientes que sofriam de } \\
\text { muita dor, além do av-MYMOP, foi } \\
\text { utilizada a escala analógica visual (VAS). }\end{array}$ & $\begin{array}{l}\text { O Reiki pareceu ser benéfico para pouco mais da } \\
\text { metade dos pacientes que o receberam. }\end{array}$ \\
\hline $\begin{array}{l}\text { THRANE } \\
\text { SE, et al., } \\
2017\end{array}$ & $\begin{array}{c}33 \text { pessoas- } \\
16 \text { crianças- } 8 \text { verbais e } 8 \text { não } \\
\text { verbais, a média de idade foi } \\
\text { de } 12,6 \text { anos (com Variações } \\
\text { de } 8 \text { a } 16 \text { anos), } \\
16 \text { mães (com idade média de } \\
43,7 \text { anos) e } 1 \text { enfermeira. }\end{array}$ & - & $\begin{array}{l}2 \text { vezes } \\
24 \text { min }\end{array}$ & $\begin{array}{l}\text { Mínimo de } \\
1 \text { e um } \\
\text { máximo de } \\
3 \text { dias } \\
\text { entre as } \\
\text { sessões. } \\
\text { SI }\end{array}$ & $\begin{array}{c}\text { Escala analógica visual (VAS) mensura } \\
\text { dor e ansiedade. • Escala de Dor de } \\
\text { Wong-Baker FACES1 (apresentada } \\
\text { simultaneamente em } 1 \text { página). • EAV } \\
\text { (padrão ouro para avaliação da dor). • } \\
\text { Children's Fear Scale (escala para } \\
\text { avaliar medo e ansiedade). • As taxas } \\
\text { cardíaca e respiratória serviram como } \\
\text { medidas substitutas para o relaxamento. }\end{array}$ & $\begin{array}{l}\text { O Reiki, pode ser benéfico para apoiar métodos } \\
\text { tradicionais de controle da dor e da ansiedade em } \\
\text { crianças que recebem cuidados paliativos. }\end{array}$ \\
\hline $\begin{array}{l}\text { CHIRICO A, } \\
\text { et al., } 2017\end{array}$ & $\begin{array}{c}55 \text { pacientes com câncer de } \\
\text { mama. } \\
\text { (A idade variou de } 23 \text { a } 65 \\
\text { anos } \\
\text { Anos) }\end{array}$ & II & $60 \min$ & SI & $\begin{array}{c}\text { Formulário Breve Inventário do } \\
\text { Comportamento do Câncer, versão } \\
\text { italiana. Os participantes relatam seu } \\
\text { nível de confiança para realizar cada } \\
\text { comportamento de enfrentamento. • } \\
\text { Perfil dos Estados do Humor (POMS). } \\
\text { Inventário de Ansiedade Traço-Estado } \\
\text { [Formulário STAI Y]. }\end{array}$ & $\begin{array}{l}\text { De uma perspectiva prática, o estudo fornece } \\
\text { resultados perspicazes para profissionais de saúde. }\end{array}$ \\
\hline
\end{tabular}

Legenda: aproximadamente; SI: Sem informação.

Fonte: Guimarães VHD, et al., 2020. 
Chirico A, et al. (2017) evidenciaram que aqueles indivíduos que receberam tratamento com Reiki tiveram redução da ansiedade e melhora do humor, inversamente, em relação aos pacientes que receberam apenas o tratamento padrão. Além disso, observou-se que os pacientes que se sentiram mais capazes de lidar com o câncer relataram maior impacto do tratamento com Reiki na redução a ansiedade.

Uma pesquisa desenvolvida em uma unidade de Câncer do Hospital das Clínicas/UNICAMP, em que 36 pessoas receberam Reiki, mais da metade dos pacientes relataram sentirem-se mais serenos e relaxados depois de receber as sessões do Reiki. Informaram ainda que se sentiram mais dispostos a enfrentar a doença e serem submetidos ao tratamento convencional.

Contudo, sua análise estatística não revelou resultados significativos. Há limitações para este estudo, em particular o tamanho da amostra e comparação dos grupos, bem como, o fato de a amostra não ter sido randomizada. Alguma das dificuldades que a equipe de pesquisa enfrentou diz respeito ao espaço físico no qual não era ideal, interferindo no trabalho (SIEGEL P, et al., 2016).

Em contrapartida, Thrane SE, et al. (2017) desenvolveram o seu estudo com crianças, avaliando a dor e ansiedade como sintomas debilitantes. Nesta pesquisa, as crianças tinham a idade média de 12,6 anos. Todos os valores médios para todos os resultados variáveis para crianças que verbalizavam e as que não verbalizavam diminuíram após tratamento com Reiki. Entretanto a pesquisa foi feita com pequena amostra, sendo necessário um estudo maior, para apoiar as tendências observadas neste estudo.

Dos 21 artigos analisados, 13 demostraram resultados positivos com o uso do Reiki como medida complementar no tratamento do câncer, colaborando para o bem-estar do paciente e para redução dos sintomas associados ao tratamento.

Ao minimizar os efeitos colaterais durante o tratamento padrão, favorece-se uma maior adesão do paciente, oferecendo uma melhor qualidade de vida a esse indivíduo. No entando, apenas um artigo mostrou que não foi possível demonstrar um resultado significativo com o uso do Reiki.

A partir dos 21 estudos, identificou-se que os trabalhos de Demir M, et al. (2015) e Orsark G, et al. (2015), apresentaram a variável pré e pós intervenção do Reiki com pelo menos um grupo controle com média e desvio padrão, sendo selecionados para análise do tamanho do efeito intergrupos.

O escore de efetividade foi avaliado por meio da escala de Hopkins W, et al. (2009) para o tamanho do efeito com e sem intervenção de Reiki. Os resultados apontaram-se de forma satisfatória na melhoria da qualidade de vida de pacientes submetidos a sessões de Reiki, conforme tabela 2 (MARCUS DA, et al., 2013).

A literatura científica demonstra o surgimento precoce do intenso sofrimento psicossocial durante 0 processo diagnóstico do câncer. Nessa fase é importante avaliar o estresse dos pacientes, a fim de aplicar uma intervenção direcionada para prevenir distúrbios psiquiátricos que persistirá em longo prazo.

Entre muitas intervenções para reduzir a ansiedade durante as fases do diagnóstico e tratamento do câncer, a Medicina Complementar Alternativa mostrou-se mais ampla nos estudos científicos projetados para avaliar seu impacto na resposta dos pacientes (ALARCÃO Z e FONSECA JR, 2016).

Post-White e colaboradores descrevem em seu estudo os efeitos da massagem terapêutica e do toque de cura, comparados ao tratamento convencional quimioterápico na indução do relaxamento físico e redução de sintomas como diminuição da dor e melhora dos estados de humor e fadiga (POST-WHITE J, et al., 2003). 
Tabela 2 - Tamanho do efeito intergrupos para variáveis de qualidade de vida em pacientes submetidos a sessões de reiki.

\begin{tabular}{|c|c|c|c|c|c|}
\hline \multirow{2}{*}{$\begin{array}{l}\text { Autor/Ano } \\
\text { Demir M, et al. }{ }^{\dagger}\end{array}$} & \multirow[t]{2}{*}{ Amostra } & \multirow[t]{2}{*}{ Pré-test } & \multirow[t]{2}{*}{ Pós-test } & \multicolumn{2}{|c|}{$\begin{array}{l}\text { Tamanho do efeito } \\
\text { intergrupos }\end{array}$} \\
\hline & & & & & \\
\hline \multirow{2}{*}{ Dor } & $C T=10$ & $3,70 \pm 2,75$ & $4,20 \pm 2,69$ & \multirow{2}{*}{0,97} & \multirow{2}{*}{ Moderado } \\
\hline & $E P=8$ & $3,62 \pm 1,06$ & $2,00 \pm 0,75$ & & \\
\hline \multirow{2}{*}{ Estresse } & CT & $4,3 \pm 2,89$ & $4,90 \pm 2,42$ & \multirow[b]{2}{*}{1,37} & \multirow{2}{*}{$\begin{array}{l}\text { Muito } \\
\text { grande }\end{array}$} \\
\hline & EP & $4,25 \pm 1,98$ & $1,37 \pm 0,74$ & & \\
\hline \multirow[b]{2}{*}{ Fadiga } & $\mathrm{CT}$ & $2,70 \pm 1,76$ & $3,80 \pm 2,25$ & \multirow{2}{*}{1,55} & \multirow{2}{*}{$\begin{array}{l}\text { Muito } \\
\text { grande }\end{array}$} \\
\hline & EP & $2,87 \pm 0,83$ & $1,75 \pm 0,70$ & & \\
\hline \multicolumn{6}{|l|}{ Orsark G, et al. ${ }^{\ddagger}$} \\
\hline \multirow{3}{*}{$\begin{array}{l}\text { Qualidade de vida específica para } \\
\text { câncer de mama }\end{array}$} & $\mathrm{CT} 1=11$ & $110,72 \pm 0,06$ & $114,48 \pm 0,07$ & \multirow{3}{*}{$\begin{array}{l}29,23^{*} \\
53,34\end{array}$} & \multirow{3}{*}{$\begin{array}{l}\text { Perfeito } \\
\text { Perfeito }\end{array}$} \\
\hline & $\mathrm{CT} 2=10$ & $99,67 \pm 0,06$ & $98,79 \pm 0,06$ & & \\
\hline & $E P=15$ & $103,36 \pm 0,05$ & $105,53 \pm 0,06$ & & \\
\hline \multirow{3}{*}{$\begin{array}{l}\text { Humor através das sessões de } \\
\text { quimioterapia }\end{array}$} & CT1 & $5,17 \pm 0,02$ & $1,76 \pm 0,01$ & \multirow{3}{*}{$\begin{array}{c}106,07^{*} \\
74,61\end{array}$} & \multirow{3}{*}{$\begin{array}{l}\text { Perfeito } \\
\text { Perfeito }\end{array}$} \\
\hline & CT2 & $4,97 \pm 0,02$ & $4,26 \pm 0,01$ & & \\
\hline & EP & $4,06 \pm 0,01$ & $2,25 \pm 0,01$ & & \\
\hline \multirow{3}{*}{ Vigor } & CT1 & $10,54 \pm 0,03$ & $9,62 \pm 0,02$ & \multirow{3}{*}{$\begin{array}{c}124,47^{*} \\
97,93\end{array}$} & \multirow{3}{*}{$\begin{array}{l}\text { Perfeito } \\
\text { Perfeito }\end{array}$} \\
\hline & CT2 & $7,31 \pm 0,03$ & $5,71 \pm 0,03$ & & \\
\hline & $\mathrm{EP}$ & $7,59 \pm 0,02$ & $3,60 \pm 0,02$ & & \\
\hline \multirow{3}{*}{ Perturbação do humor } & CT1 & $47,28 \pm 0,08$ & $22,26 \pm 0,05$ & \multirow{3}{*}{$\begin{array}{c}202,5^{*} \\
1,36\end{array}$} & \multirow{3}{*}{$\begin{array}{l}\text { Perfeito } \\
\text { Grande }\end{array}$} \\
\hline & СT2 & $52,78 \pm 0,10$ & $43,84 \pm 0,05$ & & \\
\hline & EP & $38,28 \pm 0,08$ & $29,46 \pm 0,04$ & & \\
\hline \multirow{3}{*}{ Fadiga } & CT1 & $4,12 \pm 0,01$ & $2.63 \pm 0,02$ & \multirow{3}{*}{$\begin{array}{c}103,0^{*} \\
37,3\end{array}$} & \multirow{3}{*}{$\begin{array}{l}\text { Perfeito } \\
\text { Perfeito }\end{array}$} \\
\hline & CT2 & $6,57 \pm 0,02$ & $6,66 \pm 0,02$ & & \\
\hline & EP & $6,10 \pm 0,01$ & $5,64 \pm 0,01$ & & \\
\hline
\end{tabular}

Legenda: †Formulário Patient characteristiv, ${ }^{\ddagger}$ Avaliação da qualidade de vida utilizando-se os questionários: Patient Characteristics; Escala de Avaliação Funcional de Terapia do Câncer: Câncer de Mama Versão 4, 17 (qualidade de vida multidimensional); estado do humor: Symptom Distress Scale. e Perfil dos Estados de Humor - Questionário de Forma Curta. *Grupo experimental (Reiki) comparado ao controle 1 (CT1) ou comparado ao controle 2 (CT2).

Fonte: Guimarães VHD, et al., 2020.

Os resultados demonstram que as terapias energéticas, tanto a massagem terapêutica, quanto o toque de cura reduziram a pressão arterial, a frequência respiratória e a frequência cardíaca, isoladamente a massagem terapêutica foi responsável por diminuir a pressão arterial e o toque de cura a fadiga, ambos os tratamentos a perturbação total do humor. Contudo a diminuição da perturbação total do humor ao longo do tempo em todos nos grupos de intervenção sugere que a presença de um profissional de cuidados tem alguma influência substancial na melhora do humor. Em síntese, não foi apenas a presença do terapeuta que influenciou os resultados físicos e emocionais, mas o toque real e as intervenções de cura (POST-WHITE J, et al., 2003).

Um estudo comparativo entre que pacientes que receberam tratamento tradicional com repouso e pacientes tratados de maneira convencional mais a terapia do Reiki, demonstrou que os pacientes submetidos ao tratamento energético apresentaram melhor controle da dor nos dias 1 e 4 após o tratamento, quando comparados ao grupo repouso, uma melhora na qualidade de vida também foi observada. Contudo esta melhora pode ser atribuída à presença do terapeuta nas sessões de Reiki e ausência do mesmo, durante o tratamento convencional associado ao repouso, dessa forma essa é uma possível explicação para os resultados positivos do presente estudo que corrobora com as observações do estudo de Post-White e colaboradores, que observou uma melhora no estado físico e emocional dos pacientes atribuído a presença do terapeuta (HOPKINS W, et al., 2009). 
O toque terapêutico (TT) tem como vantagens o baixo custo, não ser invasivo, não requer prescrição médica, além de não ter contraindicações. Para aplicá-lo, faz-se necessária formação em cursos de treinamento. Além disso, em diferentes estudos, o TT mostrou controlar arritmias e pressão arterial, além de aumentar o nível de hemoglobina, melhorar o sistema imunológico, acelerar o processo de cicatrização de feridas e ossos fraturados e melhorar a mobilidade em pacientes com artrite. Essas vantagens foram observadas no TT que diminuiu as náuseas em pacientes submetidos à quimioterapia, em decorrência de câncer de mama. Houve efeito significativo no tempo e frequência dos episódios de náuseas. Outro sim, mostrou-se eficaz no controle da ansiedade (VANAKI Z, et al., 2016).

Resultados apontam para a hipótese de que três tratamentos com Toque Terapêutico sem contato aumentam a sensação de bem-estar em pessoas com câncer terminal. Os achados do estudo corroboram ambas as hipóteses: pessoas com câncer terminal submetidas a toque terapêutico têm uma sensação de bem-estar maior do que aquelas que participam de um período de descanso, e a sensação de bem-estar aumenta após intervenções terapêuticas por toque. Porém ao analisar este resultado é necessário ter prudência, uma vez que, a amostra do estudo foi pequena $(n=20)$.

Contudo um dos principais fatores que explicam esses resultados positivos pode ter sido a frequência e a duração dos tratamentos com toques terapêuticos. Administrados em uma série de três tratamentos com duração entre 15,1 e 19,4 minutos (YOUNUS J, et al., 2015).

Concomitante às terapias complementares (CAM), terapia de resposta de relaxamento (TRS) e terapia de Reiki, em um estudo utilizando homens em tratamento com radioterapia externa (EBRx) para câncer de próstata, demonstrou uma melhora significativa na sub escala de bem-estar emocional da escala de qualidade de vida no grupo TRS em comparação ao grupo do Reiki. Participantes classificados como "ansiosos" no início do estudo, demonstraram uma melhora significativa no grupo TRS, e uma tendência positiva foi encontrada no grupo Reiki. Este estudo relatou a viabilidade da realização de terapias CAM em homens submetidos à EBRx para câncer de próstata. A terapia de resposta ao relaxamento melhorou o bemestar emocional e aliviou a ansiedade nos participantes. O Reiki também teve um efeito positivo em pacientes ansiosos. Como elucidado na literatura o toque terapêutico (TT) é conhecido por seus efeitos consideráveis sobre a ansiedade (CHARLESWORTH E, et al., 2018; FLEISHER KA, et al., 2014).

Diante do exposto, um estudo utilizando mulheres saudáveis submetidas a três sessões mensais consecutivas de toque terapêutico, relatou uma melhora expressiva na redução do distúrbio do humor e na redução da tensão autor referida, sendo, dessa forma, efetiva como técnica de relaxamento, redução da confusão, ansiedade e maior vigor (IACOROSSI L, et al., 2017).

Em conjunto, análises bioquímicas de urina pré e pós-teste, foram realizadas e os resultados apontam para uma diminuição significativa nos níveis de óxido nítrico, após terceira sessão de TT (IACOROSSI L, et al., 2017). Isto tem importante relevância para pacientes com câncer submetidos à quimioterapia, uma vez que, a elevação de Óxido Nítrico (ON), está relacionado à dor de cabeça, vômito e náuseas. Os resultados deste estudo têm implicações importantes sobre a redução do sofrimento e sintomas do câncer em pacientes submetidos à quimioterapia (IACOROSSI L, et al., 2017).

Os benefícios encontrados pela aplicação do Reiki em diferentes tipos de câncer demonstraram ser efetivo em diferentes parâmetros de qualidade de vida aqui avaliados (Figura 2). Contudo, as abordagens metodológicas dos estudos apresentados, demonstraram um alto grau de variabilidade entre si, dificultando assim, o estabelecimento de comparação entre as mesmas. O estabelecimento e formação de grupos intervencionais adotaram critérios e parâmetros diferentes para cada estudo avaliado, além de falta de descrição de testes de distribuição dos participantes entre grupos, bem como ausência de detalhamento dos processos de randomização e cegamento. $O$ fator limitante que pode ser encontrado é o uso de diferentes questionários para acesso à qualidade de vida, principalmente se levarmos em consideração seu caráter subjetivo. 
Figura 2 - Principais achados na melhora da qualidade de vida de pacientes diagnosticados com câncer submetidos a sessões de terapia de Reiki.

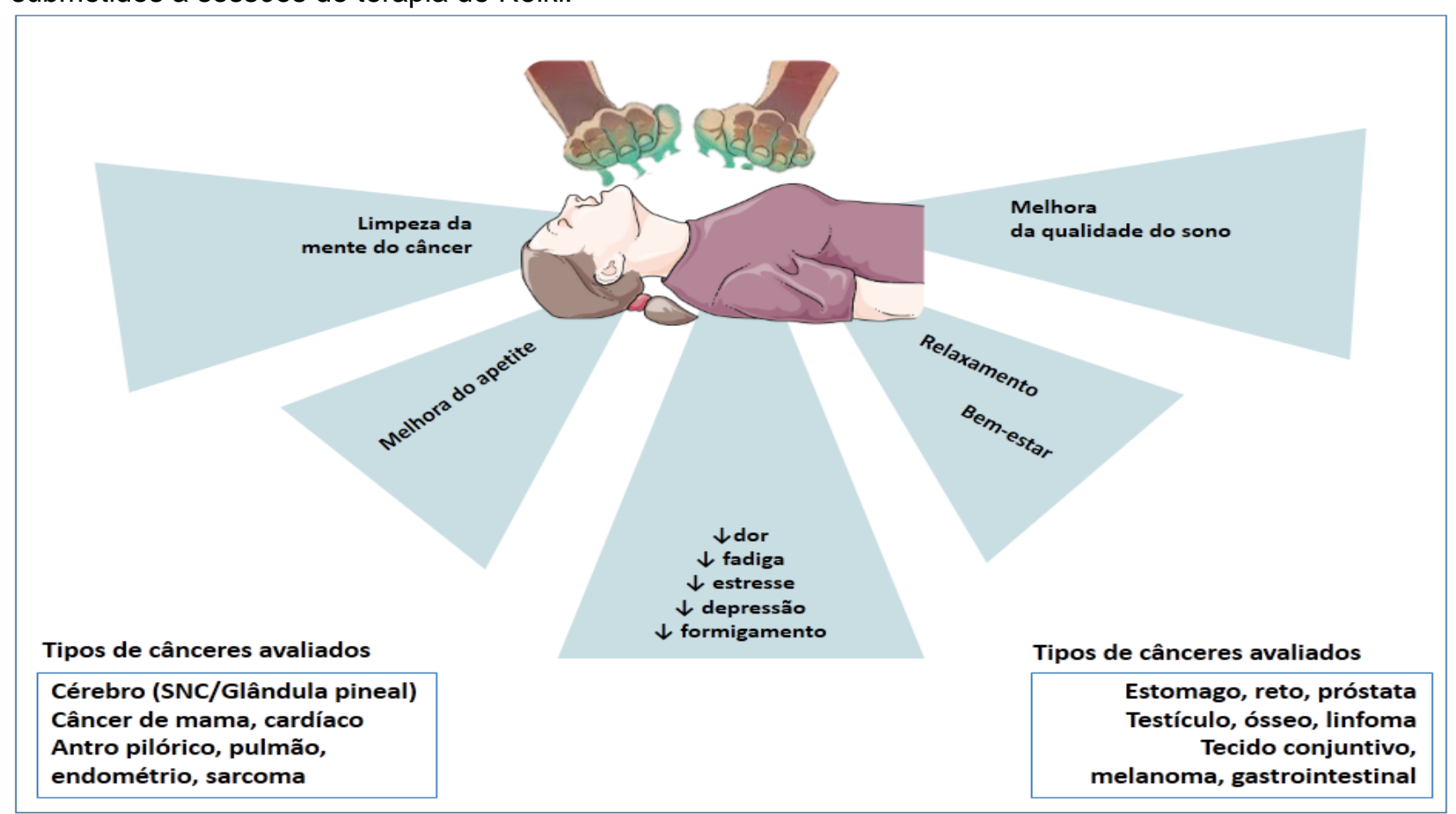

Fonte: Guimarães VHD, et al., 2020.

Além disso, o uso de termos diferentes que se referem em suma ao mesmo tipo de tratamento, tais como toque terapêutico, imposição de mãos ou Reiki são empecilhos na comparabilidade dos resultados e na busca de artigos, tendo em vista que dispõem de técnicas similares e com embasamentos de mesma raiz, apresentam particularidade da aplicação da técnica, o que pode comprometer generalizações. A avaliação dos efeitos do tratamento proposto em diversos tipos de câncer e o baixo volume de artigos que abordassem seus efeitos em um único tipo de câncer é mais um entrave à comparabilidade. Embora possam ser percebidas limitações no estudo, é possível perceber o papel benéfico da terapia em pacientes com câncer na melhora da qualidade de vida. Novas abordagens se fazem necessárias para a compreensão dos efeitos da terapia de Reiki na melhora do prognóstico do câncer.

\section{CONSIDERAÇÕES FINAIS}

O Reiki é um método alternativo que auxilia na melhora dos sintomas como relaxamento, alivio da dor, redução da ansiedade, fadiga, estresse, além aumentar o vigor e humor. A intervenção com o Reiki durante a quimioterapia reduziu os efeitos colaterais em pacientes com diferentes tipos de câncer. Dessa forma, o Reiki pode ser uma ferramenta benéfica na autogestão dos problemas de qualidade de vida em pacientes com câncer, uma vez que, a terapia é capaz de responde às necessidades emocionais dos pacientes. Assim, a implementação de um programa integrativo de Reiki mostra-se promissor como um componente do cuidado de suporte para pacientes com câncer. Portanto, o presente estudo pode contribuir para tomada de decisões na incorporação da prática complementar em pacientes diagnosticados com câncer para a melhor qualidade de vida mediante as queixas físicas e psicológicas.

\section{REFERÊNCIAS}

1. ALARCÃO Z, FONSECA JR. The effect of Reiki therapy on quality of life of patients with blood cancer: results from a randomized controlled trial. European Journal of Integrative Medicine. 2016;8(3):239-49. 
2. BEARD C, et al. Effects of complementary therapies on clinical outcomes in patients being treated with radiation therapy for prostate cancer. Cancer. 2011;117(1):96-102.

3. BIROCCO N, et al. The effects of Reiki therapy on pain and anxiety in patients attending a day oncology and infusion services unit. American Journal of Hospice and Palliative Medicine®. 2012;29(4):290-4.

4. BLOOM JR. Surviving and thriving? Psycho-Oncology: Journal of the Psychological, Social and Behavioral Dimensions of Cancer. 2002;11(2):89-92.

5. CHARLESWORTH E, et al. Complementary therapy for people with cancer; the patient's perspective. European Journal of Integrative Medicine. 2018;17:26-32.

6. CHIRICO A, et al. Self-efficacy for coping with cancer enhances the effect of reiki treatments during the Pre-surgery phase of breast cancer patients. Anticancer research. 2017;37(7):3657-65.

7. DEMIR M, et al. Effects of distant reiki on pain, anxiety and fatigue in oncology patients in turkey: a pilot study. Asian Pac J Cancer Prev. 2015;16(12):4859-62.

8. FINEOUT-OVERHOLT E, et al. Evidence-based practice step by step: Critical appraisal of the evidence: part I. AJN The American Journal of Nursing. 2010;110(7):47-52.

9. FLEISHER KA, et al. Integrative Reiki for cancer patients: a program evaluation. Integrative cancer therapies. 2014;13(1):62-7.

10. GIASSON M, BOUCHARD L. Effect of therapeutic touch on the well-being of persons with terminal cancer. Journal of Holistic Nursing. 1998;16(3):383-98.

11. HOPKINS W, et al. Progressive statistics for studies in sports medicine and exercise science. Medicine+ Science in Sports+ Exercise. 2009;41(1):3.

12. IACOROSSI L, et al. The impact of Reiki on side effects in patients with head-neck neoplasia undergoing radiotherapy: a pilot study. Professioni infermieristiche. 2017;70(3):214-21.

13. JOYCE J, HERBISON GP. Reiki for depression and anxiety. The Cochrane Library. 2015.

14. KIRSHBAUM MN, et al. An exploratory study of Reiki experiences in women who have cancer. International journal of palliative nursing. 2016;22(4):166-72.

15. LAFRENIERE KD, et al. Effects of therapeutic touch on biochemical and mood indicators in women. The Journal of Alternative and Complementary Medicine. 1999;5(4):367-70.

16. LIBERATI A, et al. The PRISMA statement for reporting systematic reviews and meta-analyses of studies that evaluate health care interventions: explanation and elaboration. PLoS medicine. 2009;6(7):e1000100.

17. MARTIN TA, et al. Cancer invasion and metastasis: molecular and cellular perspective. In Madame Curie Bioscience Database [Internet]. Landes Bioscience. 2013.

18. MATHERS CD, et al. Global burden of disease in 2002: data sources, methods and results. Geneva: World Health Organization. 2003;54.

19. MARCUS DA, et al. Symptomatic improvement reported after receiving Reiki at a cancer infusion center. SAGE Publications Sage CA: Los Angeles, CA; 2013.

20. OLSON K, et al. A phase II trial of Reiki for the management of pain in advanced cancer patients. Journal of pain and symptom management. 2003;26(5):990-7.

21. ORSAK G, et al. The effects of Reiki therapy and companionship on quality of life, mood, and symptom distress during chemotherapy. Journal of evidence-based complementary \& alternative medicine. 2015;20(1):20-7.

22. PARKER PA, et al. Psychosocial and demographic predictors of quality of life in a large sample of cancer patients. Psycho-Oncology: Journal of the Psychological, Social and Behavioral Dimensions of Cancer. 2003;12(2):183-93.

23. POHL G, et al. "Laying on of hands" improves well-being in patients with advanced cancer. Supportive care in cancer. 2007;15(2):143-51.

24. POPAT S, et al. Systematic review of microsatellite instability and colorectal cancer prognosis. Journal of clinical oncology. 2005;23(3):609-18.

25. POST-WHITE J, et al. Therapeutic massage and healing touch improve symptoms in cancer. Integrative cancer therapies. 2003;2(4):332-44.

26. RAND WL. Reiki: The healing touch: Vision Publications; 1995.

27. SCHARDT $\mathrm{C}$, et al. Utilization of the PICO framework to improve searching PubMed for clinical questions. BMC Medical Informatics and Decision Making. 2007;(7)16.

28. SIEGEL P, et al. Reiki for Cancer Patients Undergoing Chemotherapy in a Brazilian Hospital. Holistic nursing practice. 2016;30(3):174-82.

29. THRANE SE, . Reiki therapy for symptom management in children receiving palliative care: a pilot study. American Journal of Hospice and Palliative Medicine®. 2017;34(4):373-9.

30. TSANG KL, et al. Pilot crossover trial of Reiki versus rest for treating cancer-related fatigue. Integrative cancer therapies. 2007;6(1):25-35.

31. VANAKI Z, et al. Therapeutic touch for nausea in breast cancer patients receiving chemotherapy: Composing a treatment. Complementary therapies in clinical practice. 2016;22:64-8.

32. VENÂNCIO JL. Importância da atuação do psicólogo no tratamento de mulheres com câncer de mama. Revista brasileira de cancerologia. 2004;50(1):55-63.

33. YOUNUS J, et al. A case-control, mono-center, open-label, pilot study to evaluate the feasibility of therapeutic touch in preventing radiation dermatitis in women with breast cancer receiving adjuvant radiation therapy. Complementary therapies in medicine. 2015;23(4):612-6. 